\title{
A schema for common cents
}

\author{
DAVID C. RUBIN and THEDA C. KONTIS \\ Duke University, Durham, North Carolina 27706
}

\begin{abstract}
Drawings from memory of a penny, nickel, dime, and quarter were obtained from 125 undergraduates. The modal recalls for the heads of all four coins were identical, suggesting that a schema for coins exists. In an attempt to have the schema produced directly, another 75 students designed either a 2-, 7-, or 20-cent piece that would fit into our present currency. The modal drawing for this task was identical to the modal recalls. The schema for the heads derived from these experiments differs from existing coins and is not an average of them.
\end{abstract}

Coins have been used by psychologists to demonstrate a wide range of phenomena from the observation that mere exposure is not sufficient to produce a stimulus dimension (Lashley \& Wade, 1946), or much retrievable knowledge of any kind (Nickerson \& Adams, 1979), to the observation that changes in the design of coins that occurred over centuries could be simulated in the laboratory by using serial recall (Ward, 1949). Here coins will be used in one of a series of studies of recall of naturally occurring learning situations. These studies follow from the observation that most nonlaboratory remembering is of material presented many times, incidentally, in the distant past, whereas most of psychology's data are from material presented once, under intentional learning conditions, in the recent past. Thus, if psychology is to generalize to the everyday use of memory, it must expand its data base. The problem is how to do so while still obtaining quantitative, interpretable results. The solution used previously for other recall tasks and proposed here is to make use of a naturally occurring learning situation, altering the response measure to fit the available recall data.

In previous research, this same basic approach was successful with memory for word names (Rubin, 1975), prose (Rubin, 1977), semantic domains (Rubin \& Olson, 1980), and autobiographical episodes (Rubin, 1982). In these cases and the present case, recall was for material learned in the distant past, often incidentally by multiple exposure. In these cases and the present case, the amount recalled usually varied greatly from subject to subject, whereas the response measures used were much more stable and revealing of underlying cognitive processes. In fact, the data were often regular enough to allow individual subjects' behavior to be studied. The response measures used were more complex than amount recalled and had to be chosen to capture the regularity

Reprints are available from David C. Rubin, Psychology Department, Durham, North Carolina 27706. We wish to thank Susan Havrilesky for the figures, Gregory Kimble, Gregory Lockhead, and Richard Shiffrin for their comments, and Gregory Lockhead for the suggestion that we examine relations among recalled items. present in the data, varying in the four studies cited from transition frequencies to Guttman scaling (Kenny \& Rubin, 1977) to Bousfield clustering (Bousfield \& Bousfield, 1966) to the distribution of memories over time with the amount recalled by each subject held constant. Nonetheless, the measures used were all within the standard repertoires of verbal learning and cognitive psychology.

The underlying assumption that allows this work to proceed is that the complex natural environment often constrains people more than the simpler laboratory setting. That is, the more complex, organized stimulus situation often produces the more regular behavior. Such is the case, for instance, when one compares lists of words to prose (Rubin, 1978, 1980). The results to date are optimistic in that they indicate that, with some informative reservations, the basic findings of highly controlled studies will generalize to less controlled situations.

Before returning to the study of coins, some familiarity with the stimuli to be used here will be helpful. Although most readers have seen the heads of U.S. coins many times in their lives, most will not know what they look like (Nickerson \& Adams, 1979). Figure 1 is provided to save those readers from fumbling in their pockets. The heads in Figure 1 and on all current U.S. coins have clearly visible: a profile, a date, "Liberty," and "In God We Trust." On actual heads, there may be a mint mark below the dates and the artist has discreetly signed his work. Nothing else appears on the head of any current coin. Also, note that the position of the inscriptions varies from coin to coin, as does the style. The central profile could provide a focus around which a schema for coins could be organized. The diversity in the placement of the inscriptions insures that if a single schema exists for all coins, it should be easily distinguishable with respect to the placement of inscriptions from most of the actual coins, as they are easily distinguishable from each other.

The opposite is true for the tails. The central figures of the tails are visually and semantically quite distinct from each other, whereas the inscription placement is quite similar. In particular, the penny, dime, quarter, half 


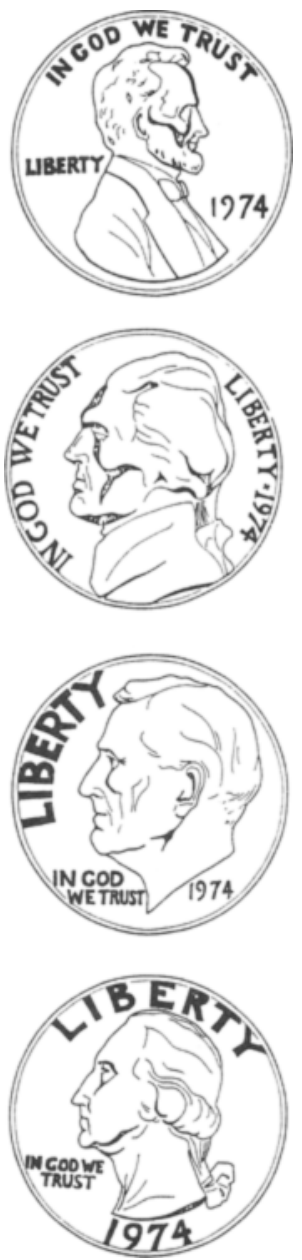

Figure 1. Actual coins now in use.

dollar, Eisenhower dollar, and Susan B. Anthony dollar all have "United States of America" on the top rim, a value on the bottom rim, and "E Pluribus Unum" placed symmetrically but not on a rim. Thus, unlike the heads, for the tails there is no clear central figure to serve in a schema, and more seriously, it would be extremely difficult to tell a schema from the actual coins with respect to the placement of inscriptions unless the schema were different from all the existing coins just listed.

\section{EXPERIMENT 1 : FREE RECALL}

\section{Method}

Subjects. An introductory psychology class of 125 undergraduates participated during part of a regularly scheduled class period.

Material. Each subject was given an $8.5 \times 11$ in. picce of paper on which were drawn two columns of four 4.5 -cm-diameter circles. The columns were labeled $H$ (for heads) and $T$ (for tails). The four circles in each column were labeled $1,5,10$, and 25 (for the four values of coins to be drawn).

Procedure. The subjects were asked to fill in the eight circles, in any order, as accuratcly as they could with the corresponding
U.S. coin. It was stressed to the subjects that the experimenter was interested in assessing their knowledge of the coins, but that artistic ability was not being judged. Subjects were encouraged to record all details and writing that are present on the actual coins in as close to the correct positions as possible.

\section{Results}

In this and the following experiment, coins were divided into sections consisting of the center area, four quadrants $(1: 30,4: 30,7: 30,10: 30$, using clockface notation), and four strips along the top, bottom, left, and right of the rim. The quadrants contained the area not covered by the rim or center. The $4: 30$ and $7: 30$ quadrants were split into the upper two-thirds and the lower one-third to differentiate between the correct position of the date on the penny and the date on the dime. In addition, a 6:00 "quadrant" was used for inscriptions placed centered in the bottom half of the coin, but not on the rim. These 12 locations were sufficient to specify the location of almost all of the recalled items, with remaining locations classified as "other." All items recalled were recorded for each subject and coin with respect to these locations. In addition, the direction of gaze of the face on the coin and the object(s) placed on the tail were recorded. Synonyms (e.g., 1 cent and penny) were combined under the most popular term, but considering each term as a separate verbatim item led to equivalent results.

The frequency of each item-location pair summed over all 125 subjects is given in Table 1 . The figures drawn are analyzed in Table 2 . For each of the four coins, the six inscriptions that actually appear on all U.S. coins are listed, as well as an "other" heading. The first three inscriptions appear on all heads. The second three inscriptions appear on all tails. The number of times an item was recalled in each position on the heads is recorded and followed by the frequency of occurrence on the tails. Of note are the observations that the recall and placement of items were far from random, with each inscription tending to be recalled in one position, independent of which of the four coins was being drawn, and with some inscriptions being recalled much more often than others, although not necessarily on the right side or location.

Given these recall results, a modal coin was constructed for the head of each value in the following manner. The head was placed on the coin facing in the most popular direction. As a head was almost always recalled in the center, other items were not recalled in the center. Thus, the placement of the head in the modal coin did not interfere with the placement of other recalled items. As it was impossible to judge which patriot was actually drawn on most recalls, the correct patriot's profile was used. Next, three additional items were placed on the head of the modal coin, as there are three on each actual coin. The most popular item. position cell from Table 1 was used first. The most popular item-position cell remaining that did not use an item or position already taken was used for the second 
Table 1

The Number of Times Items are Recalled in Particular Positions on the Heads (H) and Tails (T) of Coins (C)

\begin{tabular}{|c|c|c|c|c|c|c|c|c|c|c|c|c|c|c|c|c|c|c|c|c|c|c|c|c|}
\hline \multirow[b]{4}{*}{$\mathrm{C}$} & \multicolumn{24}{|c|}{ Position } \\
\hline & \multicolumn{14}{|c|}{ Quadrant } & \multicolumn{10}{|c|}{$\operatorname{Rim}$} \\
\hline & \multicolumn{2}{|c|}{$1: 30$} & \multicolumn{2}{|c|}{$4: 30 u$} & \multicolumn{2}{|c|}{$4: 301$} & \multicolumn{2}{|c|}{$6: 00$} & \multicolumn{2}{|c|}{$7: 30 u$} & \multicolumn{2}{|c|}{$7: 301$} & \multicolumn{2}{|c|}{$10: 30$} & \multicolumn{2}{|c|}{ Top } & \multicolumn{2}{|c|}{ Right } & \multicolumn{2}{|c|}{ Bottom } & \multicolumn{2}{|c|}{ Left } & \multicolumn{2}{|c|}{ Other } \\
\hline & $\mathrm{H}$ & $\mathrm{T}$ & $\mathbf{H}$ & $\mathrm{T}$ & $\mathbf{H}$ & $\mathbf{T}$ & $\mathbf{H}$ & $\mathrm{T}$ & $\mathrm{H}$ & $\mathrm{T}$ & $\mathrm{H}$ & T & $\mathrm{H}$ & $\mathrm{T}$ & $\mathrm{H}$ & $T$ & $\mathrm{H}$ & $\mathbf{T}$ & $\mathrm{H}$ & $\mathbf{T}$ & $\mathbf{H}$ & $\mathrm{T}$ & $\mathrm{H}$ & $\mathrm{T}$ \\
\hline \multicolumn{25}{|c|}{ Date } \\
\hline 1 & & & $58 *$ & 3 & 1 & & 2 & & 9 & & 1 & & 1 & & & & 4 & & 9 & 1 & 1 & & 2 & \\
\hline 5 & 2 & & 39 & 1 & 3 & 1 & 2 & & 10 & & & & & & & & $5^{*}$ & & 8 & 1 & & & & \\
\hline 10 & 1 & & 41 & 2 & $4^{*}$ & 1 & 1 & & 7 & & 1 & & & & & 1 & 3 & & 10 & & & & 2 & \\
\hline 25 & & & & & & & 3 & & 6 & & & & & & & & & & $23^{*}$ & 2 & 1 & & & \\
\hline & & & & & & & & & & & In $\mathrm{Gc}$ & We & Tru & & & & & & & & & & & \\
\hline 1 & & & 3 & & & 1 & 1 & & 5 & & & & & & $43^{*}$ & 12 & 1 & & 8 & 9 & & & 2 & 4 \\
\hline 5 & & & 3 & & & & & & 2 & & & & & & 24 & 15 & 1 & & 5 & 7 & $3^{*}$ & 1 & & 3 \\
\hline 10 & & & 2 & & & & & 1 & 1 & & $1^{*}$ & & & & 26 & 14 & & & 5 & 5 & 1 & & 2 & 2 \\
\hline 25 & & & 4 & & & & & & $3^{*}$ & & & & & & 26 & 17 & & & 8 & 5 & & & 2 & 1 \\
\hline & & & & & & & & & & & & ber & & & & & & & & & & & & \\
\hline 1 & & & & & & & & & $4^{*}$ & & & & & & 1 & 1 & & & & & & & 1 & \\
\hline 5 & & & & & & & & & 2 & & & & & & 1 & & $1^{*}$ & & & & & & & \\
\hline 10 & 1 & & & & & & & 1 & & & & 1 & 1 & & & & & & & & $2^{*}$ & & & \\
\hline 25 & & & & & & & & & 1 & & & & & & $3^{*}$ & & & & & & & & & \\
\hline & & & & & & & & & & & & Jalu & & & & & & & & & & & & \\
\hline 1 & & & 5 & & & & 1 & 7 & 2 & & & & & & 5 & 18 & 1 & & 14 & $29^{*}$ & & 1 & 1 & 16 \\
\hline 5 & 1 & & 3 & & & & & $5^{*}$ & & & & 3 & 1 & 1 & 2 & 12 & & & 12 & 27 & & & & 2 \\
\hline 10 & 1 & & 3 & & & & & 2 & & & & & 1 & & 4 & 12 & & & 11 & $30^{*}$ & & & & 6 \\
\hline 25 & & & 2 & & & & & 1 & & & & & & & 7 & 8 & & & 12 & $35^{*}$ & 1 & & 1 & 7 \\
\hline & & & & & & & & & & & ed $\mathbf{S}$ & tes & of $\mathrm{A}$ & ner & & & & & & & & & & \\
\hline 1 & & & & & & & & 1 & 1 & & & & & & 13 & $14^{*}$ & & & 7 & 3 & 3 & & & 1 \\
\hline 5 & & & & & & & & 1 & & & & & & & 10 & 7 & & & 5 & 3* & 2 & & & \\
\hline 10 & & & & & & & & 1 & & & & & & & 9 & $14^{*}$ & & & 5 & 4 & 1 & & & \\
\hline 25 & & & & & & & & 1 & & & & & & & 9 & $13^{*}$ & & & 4 & 5 & 1 & & 1 & \\
\hline & & & & & & & & & & & E Plu & bus & Unt & & & & & & & & & & & \\
\hline 1 & 1 & & 2 & & & & & 3 & 3 & & & & & & & 13 & & & 5 & 7 & & & 1 & $2^{*}$ \\
\hline 5 & 1 & & & & & & 2 & 2 & 1 & & & & & & & $14 *$ & & & 3 & 3 & & & & \\
\hline 10 & 1 & & & & & & & $2^{*}$ & 1 & & & & & & & 9 & & & 2 & 3 & & & & 3 \\
\hline 25 & & & & & & & & 1 & 1 & & & & & & 3 & 9 & & & 3 & 5 & & & & $3^{*}$ \\
\hline & & & & & & & & & & & & the & & & & & & & & & & & & \\
\hline 1 & 1 & & 1 & & & & 3 & & & & & & & & 3 & & & & 1 & & & & 5 & \\
\hline 5 & 1 & & & & & & 1 & $5 \dagger$ & & & & & & 1 & 2 & & & & 3 & 2 & & & 3 & 5 \\
\hline 10 & 1 & & & & & & 2 & & 2 & & & & & 1 & 2 & 2 & & & 3 & 2 & & & 4 & 4 \\
\hline 25 & 1 & & & & & & 3 & & 2 & & & & & & 2 & 1 & 1 & & & 2 & & & 4 & 1 \\
\hline
\end{tabular}

*Position on actual coin. TMonticello on nickel.

Table 2

Number of Times Figures Are Recalled in Particular Positions on the Heads and Tails of Coins

Tails Drawing

\begin{tabular}{|c|c|c|c|c|c|c|c|c|c|c|c|}
\hline \multirow[b]{3}{*}{ Coin } & & & & \multicolumn{4}{|c|}{ Buildings } & \multirow{3}{*}{$\begin{array}{l}\text { Vege- } \\
\text { tation }\end{array}$} & \multirow[b]{3}{*}{ Torches } & \multirow[b]{3}{*}{ Bird } & \multirow[b]{3}{*}{ Other } \\
\hline & \multicolumn{3}{|c|}{ Head Gaze } & \multicolumn{2}{|c|}{ Columns } & \multicolumn{2}{|c|}{ No Columns } & & & & \\
\hline & Left & Right & Ahead & Dome & No Dome & Dome & No Dome & & & & \\
\hline 1 & 70 & $42 *$ & 10 & 14 & $82^{*}$ & 1 & 7 & $11^{*}$ & & 1 & 6 \\
\hline 5 & $61^{*}$ & 31 & 15 & $65^{*}$ & 9 & 5 & 23 & 1 & & & 4 \\
\hline 10 & $45^{*}$ & 30 & 18 & 2 & 8 & 10 & 3 & $23^{*}$ & $28^{*}$ & 4 & 37 \\
\hline 25 & $56^{*}$ & 23 & 21 & 1 & 4 & 25 & 1 & 1 & & $86^{*}$ & 11 \\
\hline
\end{tabular}

*Position on actual coin. 
and then the third item. The resulting four heads are given in Figure 2. With exception of the particular head and value, the same modal coin appeared for all four values. Most of the basic elements of the actual coins are present in new positions, with "Liberty" being replaced by a value. By this measure, it appears that in addition to particulars of the various coins, people have a general schema for coins that is unlike any of the individual coins themselves.

Table 1 provides additional information about the nature of the modal coin. The first six sections of the table are for inscriptions that actually occur somewhere on the head or tail of all U.S. coins. The seventh section, labeled "other," contains all recalled inscrip. tions that were not close paraphrases of the first six section headings. There were 53 "other" responses for the heads, roughly one "other" response for each nine heads drawn. Of these 53 responses, 38 were the names of presidents, many of which appeared to be used to label otherwise unrecognizable portraits. The remaining 15 responses were mostly puns or other sayings that the subjects must have known were wrong, but which were probably included because something seemed to belong in a blank space. Examples are: "Whoever," "I'm a reflection of you," "Have a nice day," and "George." None of these 15 were used by more than one subject. Thus, it appears that while the subjects may not know all the inscriptions that appear on U.S. coins, they do have a good idea what is not on the coins. The inscriptions they recall are generally correct; the place of those inscriptions are generally wrong. This finding, as well as the others reported here, is consistent with Nickerson and Adams' (1979) data for the penny.

From Tables 1 and 2, it can be seen that as with the heads, the data from the tails were far from random. The inscriptions that appear on the actual tails tended to appear more on the recalled tails, and as with the heads, few items were recalled that do not appear somewhere on the actual coins. Moreover, most of the recalled inscriptions were put where most of the actual inscriptions are, on the top or bottom rim, although the exact inscription-location pairings were somewhat noisy. The central figures of the tails are quite well known, indicating that the actual profiles of the heads might also have been accurately drawn (as done in Figure 2) if the artistic ability of the subjects had been greater. Unfortunately, as suspected earlier, the similarity among the actual tails with respect to inscription placement makes it difficult to gather evidence that would contrast recalls driven by a schema from recalls driven by accurate memory.

Two cautions must be added to the results. First, as with most research involving schemas, the schema is inferred from grouped data. It is possible, therefore, that the schema arrived at here exists in no one subject's head but is an average of many different schemas that share enough overlap to produce the modal coins. For instance, it is possible that various subjects have differ-
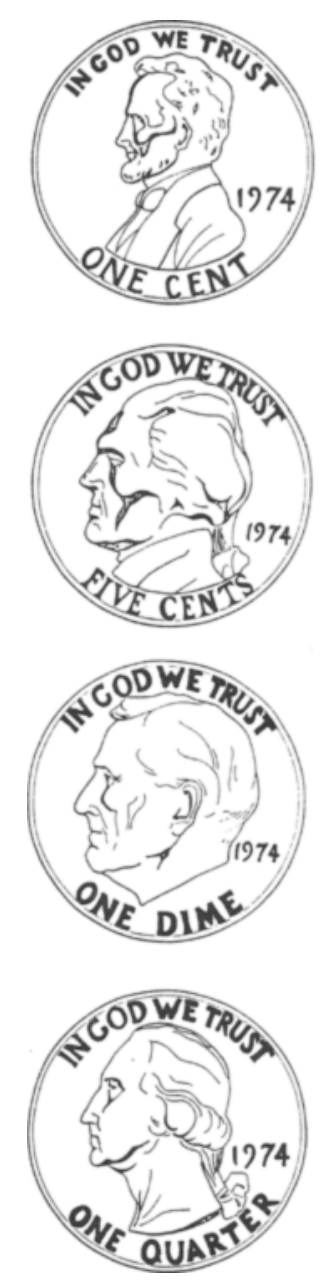

Figure 2. Modal coins constructed from recall data.

ent subsets of the four item-position pairings that go into the modal coin. Second, the mode was chosen even though it was possible that the difference between the frequency of the particular inscription-position pair chosen and another pair could have occurred by chance. A full report of the data is, therefore, presented in Tables 1 and 2 to allow alternative interpretations to be formulated. It is instructive to examine the tables to see both if other locations would be suitable for the inscriptions placed on the modal coins and if other inscriptions would be suitable for the locations used. What is most supportive of the schema notion is the striking similarity among the recalls for the four coins presented in Table 1 (and between these results and those of the next experiment). The low probability of such similarity occurring by chance and the raw distribution of responses are offered in lieu of a summary statistic because of the difficulty in using inferential statistics with repeated-measures frequency data consisting of multiple, mutually dependent responses. Complex mutual dependencies arise because each item and position tend to be used only once by each subject. 
Table 3

Location of Date as a Function of Direction of Gaze

\begin{tabular}{|c|c|c|c|c|c|c|c|c|}
\hline \multirow[b]{3}{*}{ Date } & \multicolumn{8}{|c|}{ Gaze } \\
\hline & \multicolumn{2}{|c|}{ Penny } & \multicolumn{2}{|c|}{ Nickel } & \multicolumn{2}{|c|}{ Dime } & \multicolumn{2}{|c|}{ Quarter } \\
\hline & Left & Right & Left & Right & Left & Right & Left & Right \\
\hline Left & 9 & 0 & 7 & 1 & 6 & 0 & 7 & 0 \\
\hline Right & 30 & 28 & 20 & 19 & 17 & 17 & 15 & 10 \\
\hline
\end{tabular}

Thus, the placement of an item in one position restricts the possible placement of all other items.

Another issue remains. Coins may be recalled holistically (Lockhead, 1972; Monahan \& Lockhead, 1977) instead of in parts or features. If this is the case, the relationship among the items recalled may be as important as the percentage of time that a particular item is recalled in a particular position. If visual impact is an indicator, the first place to look would be at the direction of gaze of the head. The date is the one item typically recalled in a position not symmetrical with respect to the two directions of gaze. Table 3 presents the position of the date with respect to the direction of gaze of the head. Considering each coin independently, Fisher's exact test probabilities of obtaining interactions between the direction of gaze and the position of the head as large as the ones shown are $.01, .11, .05$, and .10 , respectively.

The independent feature, or position, hypothesis offers a partial explanation of the data in Table 3: Most people put the date at the right and do so in apparent independence of the direction of gaze. For the 1-, 5-, and 10-cent coins, the right half is the correct half in which to place the date. The relational hypothesis, however, is needed to explain the observation that, with one exception, the date was only placed on the left if the head was facing left. Thus, people seem to use both positional and relational information.

\section{EXPERIMENT 2: THE SCHEMA}

The results of Experiment 1 strongly suggest that a schema for the heads of coins exists. If this is the case, people may be able to tell us what that schema is more directly than through an analysis of recall distortions. In particular, if people have a grammar for coins, they should be able to generate novel instances. That is, they should be able to draw examples of the schema itself.

\section{Method}

Subjects. Seventy-five undergraduates took part in the experiment.

Materials and Procedure. One-third of the subjects were given an $8.5 \times 11$ in. piece of paper on which were two $11.5-\mathrm{cm}$ circles and the following instructions. "In the circles below, please design a new two-cent (2申) coin which would fit into our present monetary system. Don't be creative, but rather try to follow the general schema of typical U.S. coins. Please draw both sides (heads and tails). Do not worry about your artistic ability, but please include any appropriate words or numbers on your drawings." The remaining two-thirds of the subjects were given identical forms, except that the 2-cent value was changed to 7 or 20 cents to avoid biasing the results toward any one existing coin.

\section{Results}

The inscriptions of the drawings analyzed in the same fashion as the recalls of Experiment 1 are presented in Table 4. Considering the drawings, 25 subjects had the head facing left, 19 right, and 21 ahead; on the tails, 32 subjects drew a building, 11 a statue or monument, $11 \mathrm{a}$ bird, 7 vegetation, and 17 drew other categories of objects. The results were quite similar to those of Experiment 1. In fact, the rules that produced the modal recall coin for the head in Experiment 1 produce the same modal coin here.

The relational vs. the positional analysis performed in Experiment 1 on the recall data was as follows: Of the 3 people who put the date at the left, all had the gaze

Table 4

Number of Times Items Were Drawn in Particular Positions on the Head (H) and Tail (T) of the Schema

\begin{tabular}{|c|c|c|c|c|c|c|c|c|c|c|c|c|c|c|c|c|c|c|c|c|c|c|c|c|}
\hline \multirow[b]{4}{*}{ Item } & \multicolumn{24}{|c|}{ Position } \\
\hline & \multicolumn{14}{|c|}{ Quadrant } & \multicolumn{8}{|c|}{ Rim } & & \\
\hline & \multicolumn{2}{|c|}{$1: 30$} & \multicolumn{2}{|c|}{$4: 30 u$} & \multicolumn{2}{|c|}{$4: 301$} & \multicolumn{2}{|c|}{$6: 00$} & \multicolumn{2}{|c|}{$7.30 \mathrm{u}$} & \multicolumn{2}{|c|}{$7: 301$} & \multicolumn{2}{|c|}{$10: 30$} & \multicolumn{2}{|c|}{ Top } & \multicolumn{2}{|c|}{ Right } & \multicolumn{2}{|c|}{ Bottom } & \multicolumn{2}{|c|}{ Left } & \multicolumn{2}{|c|}{ Other } \\
\hline & $\mathbf{H}$ & $\mathrm{T}$ & $\mathbf{H}$ & $\mathbf{T}$ & $\mathrm{H}$ & $\mathrm{T}$ & $\mathbf{H}$ & $\mathrm{T}$ & $\mathrm{H}$ & $\mathrm{T}$ & $\mathrm{H}$ & $\mathrm{T}$ & $\mathrm{H}$ & $\mathrm{T}$ & $\mathrm{H}$ & $\mathrm{T}$ & $\mathrm{H}$ & $\mathrm{T}$ & $\mathrm{H}$ & $\mathrm{T}$ & $\mathrm{H}$ & $\mathrm{T}$ & $\mathrm{H}$ & $\mathrm{T}$ \\
\hline Date & 1 & & $35^{*}$ & & 1 & & 5 & & 3 & & 3 & & & & & 2 & 1 & 1 & 5 & 4 & & & & \\
\hline $\begin{array}{l}\text { In God We Trust } \\
\text { Liberty }\end{array}$ & 1 & & 5 & & & & 3 & 1 & & 1 & & & 1 & & $\begin{array}{c}24^{*} \\
2\end{array}$ & 16 & 1 & 1 & 9 & $\begin{array}{l}6 \\
1\end{array}$ & 1 & 2 & 3 & 1 \\
\hline Value & 2 & 1 & 4 & & & & 3 & 6 & 3 & 1 & 1 & & 1 & 1 & 9 & 11 & & & $18^{*}$ & 13 & 1 & & 1 & 12 \\
\hline U.S.A. & & & & & & & 2 & 1 & & & & & & 1 & 17 & 11 & 1 & & 9 & 13 & & & & \\
\hline E.P.U. & & & & & & & 1 & 5 & 2 & & & & & & 4 & 11 & & & 2 & 7 & & & & 1 \\
\hline Other & 1 & & & & & & 6 & 4 & 2 & 1 & 1 & & 2 & & 5 & 1 & & 1 & 3 & 10 & & & 2 & 4 \\
\hline
\end{tabular}

*Position on modal recall coin of Experiment 1. 
to the left; of the 20 people who put the date at the right, half had the gaze to the right and half to the left. Again, results similar to the recall data were found.

The marked similarity between the data obtained from the recalls of actual coins and the data obtained from the drawings of imaginary coins suggests that people, in fact, have and use a coin schema that guides their recalls.

\section{GENERAL DISCUSSION}

People use coins daily. The results indicate that they may do so by use of a schema that is inaccurate and the same for all coins. How can this be the case? One reason is that people do not have to know what is inscribed on coins to use them properly. The coins studied here can be easily distinguished purely on the basis of size and color. Even if the color cue, which distinguishes the penny and dime, is not available because the coins are out of sight in one's pocket, the smooth vs. reeded edge cue could be used effectively. In pilot work, we found that subjects were quite good, although not perfect, at identifying U.S. coins by touch. The type of edge can be shown to be important by including foreign coins, which have different size-edge combinations, in the set to be identified. Thus, the schema of visual details described here is not needed to distinguish coins; rather, the color, size, and edge are usually enough. The schema is then a description of the limited knowledge people have of unnecessary visual details.

We would have to predict from this view that if a new coin were made with the same size, color, and edge as an existing coin even though it had a different profile, value, and design, confusion would result: a test kindly performed for us by the mint in the case of the Susan B. Anthony dollar. From the results and interpretation presented here, the similarity in size, color, and edge of the quarter and the Susan B. Anthony dollar insured that the two would be confused, even though what is imprinted on them differs considerably. Put simply, people are just not accustomed to paying attention to the visual details of coins because they have not had to in the past. Introducing a new, not clearly articulated demand to pay attention to visual details may be part of what led to the confusion and dislike for the new coin.

Another reason that a schema can be used instead of detailed information is that coins of one value are usually treated in a manner similar to coins of another value. Put in terms of current theory, coins are basic objects (Rosch, Mervis, Gray, Johnson, \& Boyes-Braem, 1976). That is, when compared to their superordinate category (money) and their subordinate categories (such as pennies and nickels), the category of coins "(a) possess a significant number of attributes in common, (b) have motor programs which are similar to one another, (c) have similar shapes, and (d) can be identified from averaged shapes of members of the class" (Rosch et al., 1976, p. 382).

Thus, there is no need for people to know what is on a coin and reason to expect that a schema exists. Given these conditions, recent work (Friedman, 1979) indicates that while individual details might not be known, a class of likely details might be known and substituted freely for the actual details. Moreover, when the actual detail is not known, some reasonable default value may be substituted (also see Brewer \& Treyens, 1981). Tables 1, 2, and 4 indicate that there are locations on coins where inscriptions are usually put, that the inscriptions used vary over a set of reasonable alternatives, and that inscriptions tend to be included even when they are not correct. That is, there exist slots that can be filled with a range of items, and these slots tend to be filled. Friedman's analysis comes from showing her subjects target pictures containing expected and unexpected objects (e.g., a toaster on a counter in a kitchen) and later testing with a range of distractors (e.g., a radio for the toaster on the counter), but her conclusions apply equally well for coins.

If the particular details of coins are not encoded or recalled, how are the particular inscriptions of the schema or prototype determined? Most researchers, at least in practice, view the prototype as an average figure around which the nonprototypical cases are perturbations (Franks \& Bransford, 1971; Posner \& Keele, 1968; Solso \& Raynis, 1979). Such a view is not helpful here.

There are several ways to average across coins. The various values could be weighted by their circulation. That is, features of coins could be counted over all coins minted (i.e., over tokens). For this case, the schema would not be found; rather, the penny would become the schema because its circulation is greater than the circulation of all other coins combined. If only the head is considered, using any method, the schema would not be found because no head has a value. Similarly, if the particular inscriptions are considered independently of their location or the coins' circulation, the schema would not be determined because all coins have a date, "Liberty," and "In God We Trust," a value, "United States of America," and "E Pluribus Unum," leaving a uniform distribution of inscriptions to choose from. A remaining alternative would be to consider the most popular inscription-location pair independently of whether it was on the head or tail. For 1978 coins, the rules used to score the data of Experiments 1 and 2 would produce a value at the bottom rim, as on the schema, "United States of America" across the top rim, replacing the "In God We Trust" of the schema, and "In God We Trust" at the 7:30 position, replacing the date at the $4: 30$ position of the schema. Using the new dollar or bicentennial coins would change the results slightly, but not the conclusions. Thus, the schema of Experiments 1 and 2 is not a physical average of existing coins under any of the averaging methods attempted.

One speculative suggestion as to the genesis of the particular schema found is that people use world knowledge that is more general than the set of stimuli being considered by the experimenter. From our knowledge 
of coins, it seems reasonable to assume that the most crucial information about a coin, which should appear clearly on its front, is its value. The left-facing profile may provide another example. It could be obtained simply by weighting each coin equally, as only the profiles on the penny and the Susan B. Anthony dollar face right (although simple weighting by circulation would produce the opposite effect). This left-facing profile on the schema and on most actual coins, however, may be a symptom of a general tendency in western civilization to have profiles face to the left (McManus \& Humphrey, 1973). That is, in general, schemas may be formed from general information and attitudes that represent more than the physical average of the stimuli they represent (Bartlett, 1932).

Most of the existing literature, including this paper, has been less than specific in defining the term schema. Four possible views are considered here. One is that a schema is an organized whole that functions in an allor-none fashion. The large proportion of subjects whose recalls were consistent with, or nearly consistent with, the modal coin but were not complete argues against this view's usefulness here. Another possibility is that a schema could be recalled incompletely but still could function as an organized whole. That is, relations among parts or features rather than the parts or features them. selves could be the functional elements. The apparent dependence of the date placement and direction of gaze provides support for this view of a schema.

A schema could also be viewed as simply a collection of independent pieces of information that vary in salience. Here the following four independent pieces would be used: (1) The value belongs at the bottom rim, (2) "In God We Trust" belongs at the top rim, (3) the date belongs at the top of the lower right-hand quadrant, and (4) the profile faces left. Along the same lines but even less organized would be a schema that combined the separate inscription and location lists at the time of recall in terms of their salience. That is, the most salient inscription not already used would be put in the most salient location not already used. Using frequency of recall as a measure of salience, the three most recalled inscriptions on the head independent of location are the date (295 responses), "In God We Trust" (182 responses), and the value (91 responses). Combining the upper and lower $4: 30$ and $7: 30$ quadrants, the most popular locations in which to place any inscription are the $4: 30$ quadrant (208 responses), the top rim (195 responses), and the bottom rim (166 responses). Pairing these two lists in order produces the modal coin. Given the high degree of agreement among subjects on where particular inscriptions should be placed, as shown in Tables 1 and 4 , and the relatively close ordering of the separate lists for inscriptions and locations, it is doubtful that this last possibility is actually the mechanism functioning here. Thus, for instance, the $4: 30$ quadrant and top rim have almost the same number of total responses in Table 1 (208 and 195, respectively), yet quite different distributions of inscriptions account for these responses.
The four independent inscription-location pieces of information coupled with enough holistic information to account for the interaction of the date and gaze is the minimal view of a schema that is necessary to adequately describe the data presented here. Which characterizations of organization should ultimately be considered as schemas and which best describe the organization in recall that is observed in domains other than coins are open questions.

\section{REFERENCES}

Bartlett, F. C. Remembering: $A$ study in experimental and social psychology. Cambridge: Cambridge University Press, 1932.

Bousfield, A. K., \& Bousfield, W. A. Measurement of clustering and of sequential constancies in repeated free recall. Psychological Reports, 1966, 19, 935-942.

Brewer, W. F., \& TrEyens, J. C. Role of schemata in memory for places. Cognitive Psychology, 1981, 13, 207-230.

Franks, J. J., \& Bransford, J. D. Abstraction of visual patterns. Journal of Experimental Psychology, 1971, 90, 65-74.

Friedman, A. Framing pictures: The role of knowledge in automatized encoding and memory for gist. Journal of Experimental Psychology: General, 1979, 108, 316-355.

KenNy, D. A., \& Rubin, D. C. Estimating chance reproducibility in Guttman scaling. Social Science Research, 1977, 6, 188-196.

LAShlEY, K. S., \& W AdE, M. The Pavlovian theory of generalization. Psychological Review, 1946, 53, 72-87.

Lockнеad, G. Processing dimensional stimuli. Psychological Review, 1972, 79, 410-419.

McManus, I. C., \& Humphrey, N. K. Turning the left cheek. Nature, 1973, 243, 271-272.

Monahan, J. S., \& Lockhead, G. R. Identification of integral stimuli. Journal of Experimental Psychology: General, 1977, 106, 94-110.

Nickerson, R. S., \& Adams, M. J. Long-term memory for a common object. Cognitive Psychology, 1979, 11, 287-307.

Posner, M. I., \& Keele, S. W. On the genesis of abstract ideas. Journal of Experimental Psychology, 1968, 77, 353-363.

Rosch, E., Mervis, C. B., Gray, W. D., Johnson, D. M., \& Boyes-Braem, P. Basic objects in natural categories. Cognitive Psychology, 1976, 8, 382-439.

Rubin, D. C. Within word structure in the tip-of-the-tongue phenomenon. Journal of Verbal Learning and Verbal Behavior, 1975, 14, 392-397.

Rubin, D. C. Very long-term memory for prose and verse. Journal of Verbal Learning and Verbal Behavior, 1977, 16, 611-621.

RuBin, D. C. A unit analysis of prose memory. Journal of Verbal Learning and Verbal Behavior, 1978, 17, 599-620.

Rubin, D. C. 51 properties of 125 words: A unit analysis of verbal behavior. Journal of Verbal Learning and Verbal Behavior, 1980, 19, 736-755.

RuBiN, D. C. On the retention function for autobiographical memory. Journal of Verbal Learning and Verbal Behavior, $1982,21,21-38$.

Rubin, D. C., \& Olson, M. J. Recall of semantic domains. Memory \& Cognition, 1980, 8, 354-366.

Solso, R. L., \& Raynis, S. A. Prototype formation from imaged, kinesthetically, and visual presented geometric figures. Journal of Experimental Psychology: Human Perception and Performance, 1979, 5, 701-712.

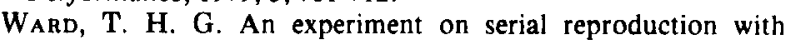
special reference to the changes in the design of early coin types. British Journal of Psychology, 1949, 39, 142-147.

(Received for publication June 11, 1982; revision accepted August 25, 1982.) 